\title{
The asteroid population in $g$-type non-linear secular resonances
}

\author{
M. Huaman, ${ }^{1 \star}$ V. Carruba, ${ }^{1 \star}$ R. C. Domingos ${ }^{2}$ and S. Aljbaae ${ }^{1}$ \\ ${ }^{1}$ School of Natural Sciences and Engineering, São Paulo State University (UNESP), Guaratinguetá, SP 12516-410, Brazil \\ ${ }^{2}$ Campus of São João da Boa Vista, São Paulo State University (UNESP), SP 13876-750, Brazil
}

Accepted 2017 April 3. Received 2017 March 31; in original form 2016 November 17

\begin{abstract}
Non-linear secular resonances of $g$-type, i.e. involving the frequency of precession $g$ of the asteroid pericentre, can affect the proper eccentricities of asteroids in resonant or near-resonant configurations. We first identified objects that could potentially be affected by non-linear secular resonances of this type. We then numerically integrated these objects and checked for their resonant argument. We identified a population of 1517 asteroids in $g-2 g_{6}+g_{5}$ librating states, and of 128 objects in $g-3 g_{6}+2 g_{5}$ resonant configurations. While secular resonances are rather extended structures and many objects from different and unrelated parts of the main belt could be encountered within, we found that $g-2 g_{6}+g_{5}$ librators are predominantly of the $\mathrm{S}$ taxonomical type (56 per cent of the total), but with a significant fraction of other spectral types. No spectral type dominates in the population of $g-3 g_{6}+2 g_{5}$ resonators. Several asteroid families are affected by the $g-2 g_{6}+g_{5}$ secular resonance. The Astraea group is cut into two by this resonance, while the Tirela and Brasilia groups are on the resonance centre and on the left side, respectively. The $g-2 g_{6}+g_{5}$ secular can significantly affect the shape of families inside the resonance, such as Astraea. It can also increase the flux of asteroids to nearby powerful mean-motion resonances, such as the $5 \mathrm{~J}:-2 \mathrm{~A}$ and the $2 \mathrm{~J}:-1 \mathrm{~A}$. As expected, the long-term effect of $g$-type resonances on inclinations is essentially negligible.
\end{abstract}

Key words: minor planets, asteroids: general-celestial mechanics.

\section{INTRODUCTION}

According to Williams (1969), secular resonances play an important role in the dynamics of small bodies in the Solar system. They can change the eccentricity and inclination of objects that were captured in librating states of these resonances, and sculpt the shape of asteroid families in their neighbourhood. Linear secular resonances occur when there is a commensurability between the precession frequency of the longitude of pericentre $g$, or of the longitude of the node $s$ of a given asteroid and the frequencies of a planet. The three main linear secular resonances in the Solar system are $v_{6}=g-g_{6}, v_{5}=g-g_{5}$ and $v_{16}=s-s_{6}$ (Kneževic et al. 1991; Morbidelli \& Henrard 1991), where $g_{5}=4.257, g_{6}=28.243$, and $s_{6}=-26.345 \operatorname{arcsec}_{\mathrm{yr}^{-1}}$. Non-linear secular resonances involve higher order combination of frequencies, as for the case of the $z_{k}=k\left(g-g_{6}\right)+s-s_{6}=k v_{6}+v_{16}(k=1,2,3 \ldots)$ secular resonances. Amongst non-linear secular resonances, $g$-type secular resonances are those that only involve the $g$ asteroidal frequency (and other planetary frequencies), as for example, the cases of the $2 v_{6}-v_{5}=g-2 g_{6}+g_{5}$ or $3 v_{6}-2 v_{5}=g-3 g_{6}+2 g_{5}$ secular resonances. The main focus of this work will be to study the dynamical evolution of asteroids interacting with $g$-type secular resonances, so as to ascertain the number of objects currently in librating states, the dynamical importance of each given resonance, especially when non-gravitational forces such as the Yarkovsky and YORP effects are accounted for, and their effect on selected asteroid families.

\section{RESONANCE DYNAMICS FOR NON-LINEAR SECULAR RESONANCES}

As a preliminary criterion to identify asteroids inside the $g$-type non-linear secular resonances, we used Carruba (2009) approach. Given the $z_{1}$ secular resonance of argument $z_{1}=g-g_{6}+s-s_{6}$, we considered as objects most likely to be in librating states those for which $g+s=\left(g_{6}+s_{6}\right) \pm 0.3 \operatorname{arcsec} \mathrm{yr}^{-1}$. Since the width of the librating region for asteroids in the fourth-order secular resonance tends to be less than $0.3 \operatorname{arcsec}_{\mathrm{yr}^{-1}}$ (Carruba 2009), we believe that this could be a good criterion to identify objects likely to be in fourth- and sixth- order $g$-type non-linear secular resonances.

For the purpose of identifying objects in the regions affected by $g$-type non-linear secular resonances, we used a catalogue of 406251 synthetic proper elements numerically computed with numbered and multiposition asteroids available at the AstDyS data base (Knežević \& Milani 2003) ${ }^{1}$. According to the theory, the asteroid 
Table 1. Number of likely and actual resonators for all $g$-type secular resonances up to order six. The first column displays the secular resonance argument as a function of linear resonance arguments. The second column presents the secular resonance argument in terms of frequencies, the third column the values of the asteroidal frequencies equivalent to the combination of the planetary frequencies and the fourth column shows the number of likely resonators. The fifth column displays the number of actual resonators (see discussion in the next section).

\begin{tabular}{|c|c|c|c|c|}
\hline $\begin{array}{l}\text { Res. argument } \\
\text { linear resonances }\end{array}$ & $\begin{array}{l}\text { Res. argument } \\
\text { frequencies }\end{array}$ & $\begin{array}{l}\text { Frequency } \\
\text { value ["/yr] }\end{array}$ & $\begin{array}{l}\text { Likely } \\
\text { resonators }\end{array}$ & $\begin{array}{l}\text { Actual } \\
\text { resonators }\end{array}$ \\
\hline \multicolumn{5}{|c|}{$g$-type, first harmonic: } \\
\hline $2 v_{5}-v_{6}$ & $g-2 g_{5}+g_{6}$ & -19.729 & 2 & 0 \\
\hline $3 v_{5}-2 v_{6}$ & $g-3 g_{5}+2 g_{6}$ & -43.715 & 2 & 0 \\
\hline $2 v_{6}-v_{5}$ & $g-2 g_{6}+g_{5}$ & 52.229 & 3293 & 1517 \\
\hline $3 v_{6}-2 v_{5}$ & $g-3 g_{6}+2 g_{5}$ & 76.215 & 694 & 128 \\
\hline $2 v_{6}-v_{7}$ & $g-2 g_{6}+g_{7}$ & 53.393 & 1867 & 24 \\
\hline $2 v_{6}-2 v_{5}+v_{7}$ & $g-2 g_{6}+2 g_{5}-g_{7}$ & 51.065 & 761 & 26 \\
\hline \multicolumn{5}{|c|}{$2 g$-type, second harmonic: } \\
\hline $3 v_{5}-v_{6}$ & $2 g-3 g_{5}+g_{6}$ & -7.736 & 5 & 0 \\
\hline $3 v_{6}-v_{5}$ & $2 g-3 g_{6}+g_{5}$ & 40.236 & 1178 & 116 \\
\hline \multicolumn{5}{|c|}{$3 g$-type, third harmonic: } \\
\hline $2 v_{5}+v_{6}$ & $3 g-2 g_{5}-g_{6}$ & 12.252 & 20 & 2 \\
\hline $2 v_{6}+v_{5}$ & $3 g-2 g_{6}-g_{5}$ & 20.248 & 58 & 2 \\
\hline $2 v_{6}+v_{7}$ & $3 g-2 g_{6}-g_{7}$ & 19.859 & 45 & 0 \\
\hline \multicolumn{5}{|c|}{$4 g$-type, fourth harmonic: } \\
\hline $3 v_{5}+v_{6}$ & $4 g-3 g_{5}-g_{6}$ & 10.254 & 13 & 0 \\
\hline $3 v_{6}+v_{5}$ & $4 g-3 g_{6}-g_{5}$ & 22.247 & 59 & 0 \\
\hline \multicolumn{5}{|c|}{$5 g$-type, fifth harmonic: } \\
\hline $3 v_{5}+2 v_{6}$ & $5 g-3 g_{5}-2 g_{6}$ & 13.851 & 38 & 0 \\
\hline $3 v_{6}+2 v_{5}$ & $5 g-3 g_{6}-2 g_{5}$ & 18.649 & 41 & 0 \\
\hline
\end{tabular}

diameters are a function of their absolute magnitude $H$ and geometric albedo $p_{v}$ through the relationship (Carruba et al. 2003):

$D=\frac{1329 \mathrm{~km}}{\sqrt{p_{v}}} \times 10^{H / 5}$,

We selected objects with absolute magnitude $H<15.5$, so as to concentrate our attention on the largest bodies. Our results are shown in Table 1 for all $g$-type secular resonances up to the order ten. Fig. 1 shows an $(a, \sin (i))$ projection of the proper elements of likely resonators, as reported in Table 1. A large number of likely resonators were located in the central main belt, between the mean-motions resonances $3 A: 1 J$ and $8 A: 3 J$. Smaller populations were found in the outer main belt and at the edges of the $2 A$ : $1 J$ mean-motion resonance. All the objects have $\sin (i)<0.5$. The names of asteroid families with members interacting with any of these resonances is also shown in the figure. We selected asteroid families from the lists from Nesvornỳ, Brož \& Carruba (2015) and Milani et al. (2014). Some families in the list from Milani et al. (2014) do not yet have a family identification number (FIN), since they do not yet fulfill the criteria from Nesvorný et al. (2015) for family identification. For instance, they may not exhibit a clear V-shape in the $(a, H)$ domain, or may be artefacts created by the way local secular dynamics affects the computation of standard synthetic proper elements. The Astraea family might be one of these cases. Nevertheless, we list these families i) for the sake of completeness, and ii) because they may be interesting for studying the dynamical evolution of asteroids in $g$-type non-linear secular resonances, which is the main goal of this paper.

For the case of $g-2 g_{6}+g_{5}$ resonance, 10 families are found close to and within the likely resonant region. Amongst these, the Astraea family with 2425 candidates, the Ikenozenni group with 88 candidates, the Bankakuko family with 53 candidates, the Harig group with 53 candidates, the Wagman family with 92 candidates, the Euphrosyne group with 5 candidates, the Brasilia family with 4 candidates, the Gefion group with 5 asteroids, and finally, the Eunomia family with 3 candidates were all identified. To better understand the importance of local dynamics in the evolution of these groups, we next obtained dynamical maps for the affected regions.

\section{DYNAMICAL MAP IN THE AFFECTED REGIONS}

We computed dynamical maps of synthetic proper elements with test particles in the region of the main belt affected by these types of resonances. We used the SWIFT-MVSF symplectic integrator from the SWIFT package (Levison \& Duncan 1994) modified by Brož (1999) to include online digital filtering. The particles were taken in an equally spaced grid with respect to the plane of the study. We used 270 by 155 particles for the $(a, \sin (i))$ plane, and 270 by 200 particles for the $(a, e)$ plane. The steps were 0.005 au in $a, 0.2$ in $i$ and 0.004 in $e$, respectively. The initial values of $\omega$, and $\lambda$ of the test particles were fixed at those of (410) Chloris (1896 CH), an asteroid with an intermediate location between these resonant regions. Synthetic proper elements $(a, e, \sin (i))$ and frequencies $(n, g, s)$ of these test particles were calculated using the approach described in Carruba (2010).

Our results are shown in Fig. 2. Overall, results of dynamical maps are in agreement with those of our previous analysis of likely resonators. By affecting the values of $g$ asteroidal frequencies, mean-motion resonances also affect the behaviour of $g$-type secular resonances. This is particularly visible near the $3 \mathrm{~J}$ :-1 A mean-motion resonance, where we observe an exponential-like behaviour of the orbital location of likely resonators in the $g+2 g_{6}-g_{5}$ resonance in the proximity of this resonance (see Fig. 2). 


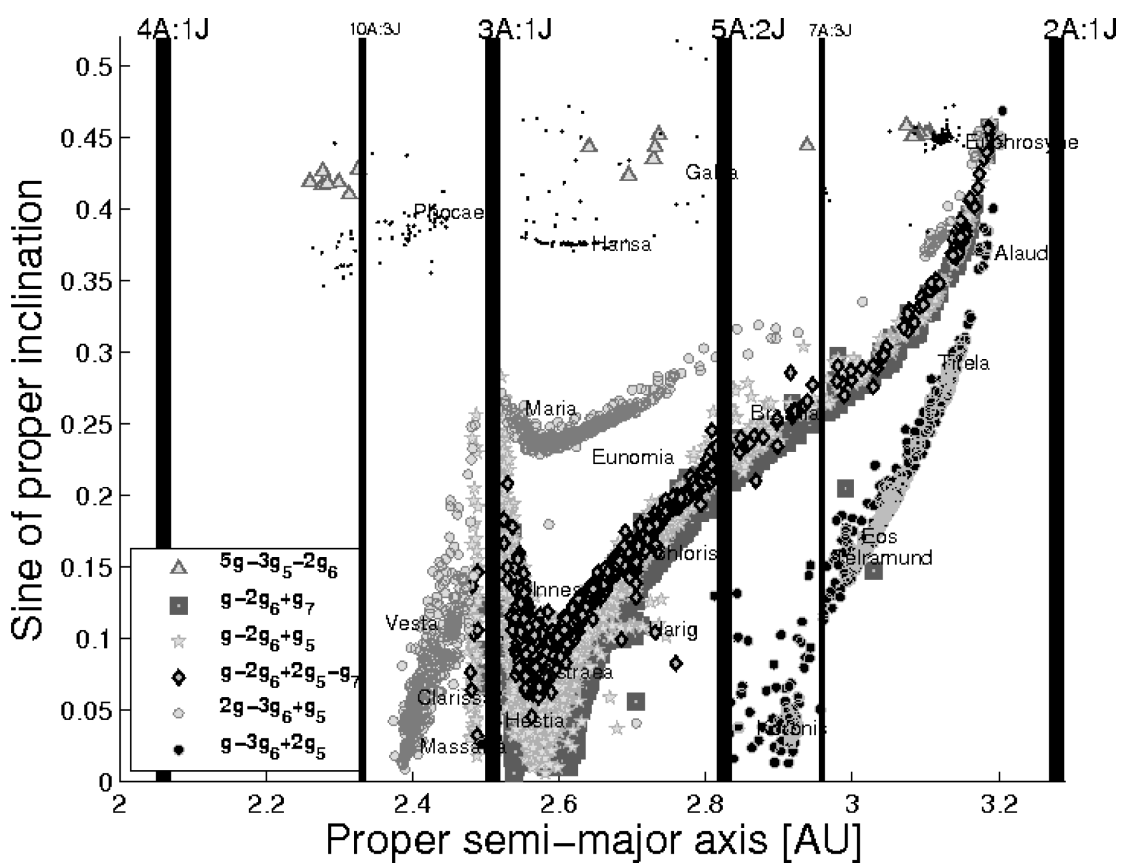

Figure 1. An $(a, \sin (i))$ projection of the likely resonators inside the regions affected by the non-linear secular resonance of $g$-type in the main belt. Black lines display the mean-motion resonance locations. Marked stars, black circles, grey circles, squares, diamonds, triangles and black dots are associated with the orbital location of likely resonators of the $g-2 g_{6}+g_{5}, g-3 g_{6}+2 g_{5}, 2 g-3 g_{6}+g_{5}, g-2 g_{6}+g_{7}, g-2 g_{6}+2 g_{5}-g_{7}$ and $5 g-3 g_{5}-2 g_{6}$ non-linear secular resonances, respectively.
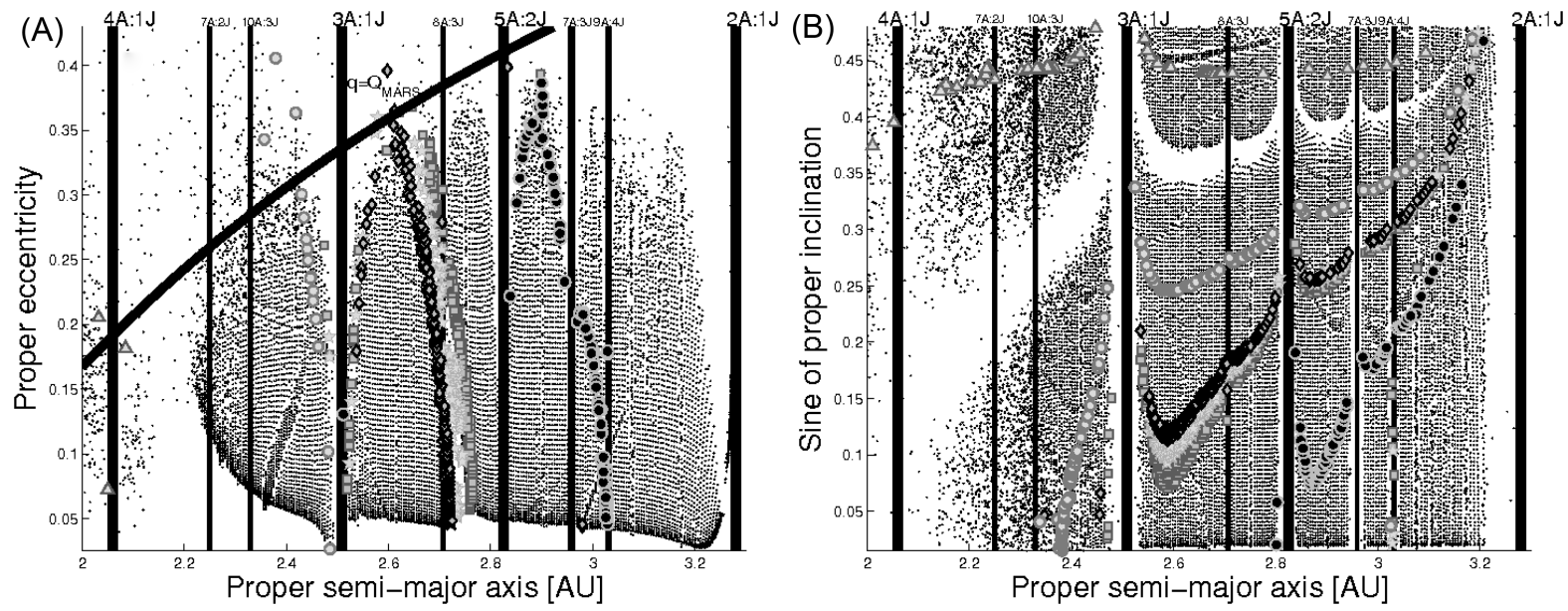

Figure 2. Dynamical maps in the $(a, e)($ Panel A) and $(a, \sin (i))($ Panel B) planes, in the main belt. Black dots display the orbital location, the proper elements for the test particles; and vertical black lines display the main mean-motion resonances. The same symbols as in Fig. 1 were used to identify the resonant regions affected by non-linear secular resonance of $g$-type.

\section{SECULAR DYNAMICS FOR LIKELY RESONATORS}

As previously discussed, not all likely resonators will actually be in librating states of non-linear secular resonances. Some will be in circulating orbits, while others may alternate phases of circulation and librations. To better identify the dynamical importance of each resonance, we performed numerical simulations with the likely resonators identified in Section 2, and checked the time behaviour of the resonant argument of each given resonance. For example, in the case of $g-2 g_{6}+g_{5}$ we have $\sigma=\varpi-2 \varpi_{6}+\varpi_{5}$. We then used the criteria given by Machuca \& Carruba (2012) to identify different states of the resonant argument for orbits in states of circulation (resonant angle covering values from $0^{\circ}$ to $360^{\circ}$ ), libration (resonant angle covering a limited range of values) and switching orbits, that alternates phases of circulation and libration.

In Fig. 3, we show the state of asteroid 42095 (2001 AY25) from the Ikenozenni family, whose orbit is in a state of libration of the $g-2 g_{6}+g_{5}$ resonance (actual resonator hereafter). Panel A displays the polar diagram for the resonant elements $\left(\psi_{1}, \psi_{2}\right)$, defined as (Charlier 1902) $)^{2}$

$\psi_{1}=\sqrt{2\left(1-\sqrt{1-e^{2}}\right)} \cdot \cos \left[\varpi-2 \varpi_{6}+\varpi_{5}\right]$,

\footnotetext{
${ }^{2}$ These elements are asymptotically equal to $e \cos (\sigma)$ and $e \sin (\sigma)$ for small values of $e$
} 


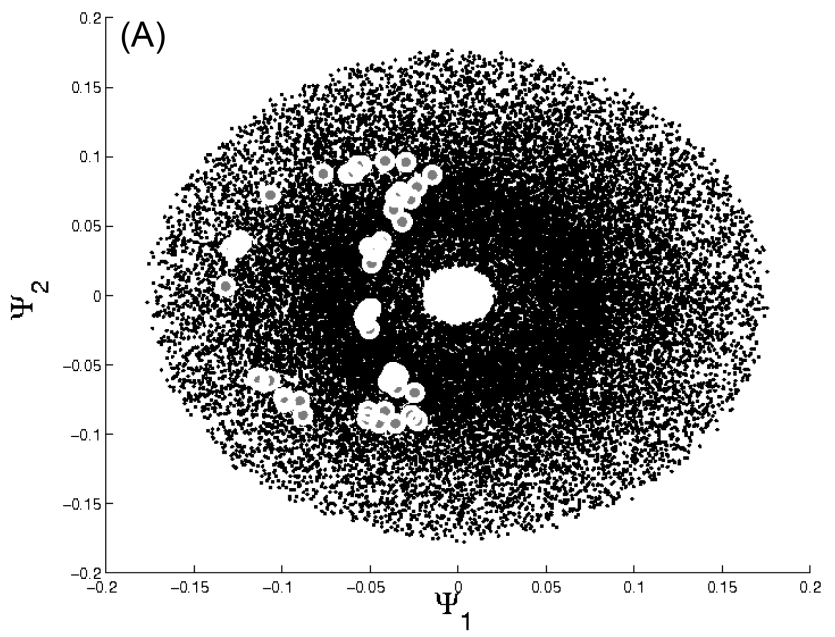

(B)

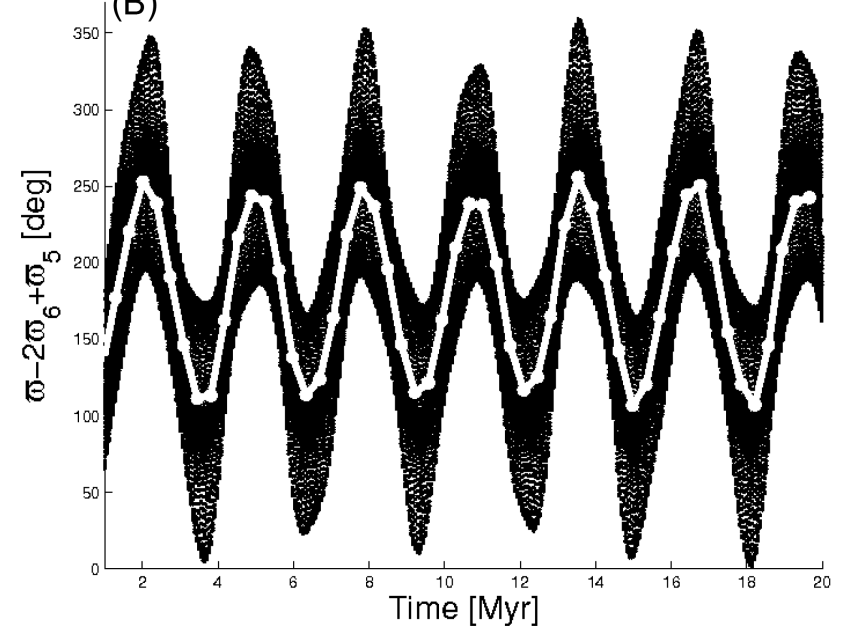

Figure 3. Panel A: orbital evolution in the polar plane $\left(\psi_{1}, \psi_{2}\right)$. Panel B: the time-evolution of the resonance argument $\sigma=\varpi-2 \varpi_{6}+\varpi_{5}$ for the same asteroid.

$\psi_{2}=\sqrt{2\left(1-\sqrt{1-e^{2}}\right)} \times \sin \left[\varpi-2 \varpi_{6}+\varpi_{5}\right]$,

where $e$ is the asteroid eccentricity. Black dots show the evolution of orbital elements filtered so as to eliminate all terms with periods of up to $600 \mathrm{yr}$, while the full circles show filtered elements without terms with periods up to $300000 \mathrm{yr}$. The first choice allows to filter all short-period terms, while the second choice allows to focus on long-period terms associated with the libration in the $g-2 g_{6}+g_{5}$ resonance. Panel B shows the time behaviour of the resonant argument $\sigma=\varpi-2 \varpi_{6}+\varpi_{5}$ for the same asteroid. The long-time behaviour of the angle, after eliminating all the short-period perturbations, is shown in white. This asteroid is in a librating state around an equilibrium point at $180^{\circ}$. Interested reader can find other examples of time behaviour of resonant arguments for asteroids in circulating and switching states in Machuca \& Carruba (2012).

For $1 g$-type resonances, we found 1517 actual resonators of the $g-2 g_{6}+g_{5}$ secular resonance, and 128 resonators of the $g-3 g_{6}+2 g_{5}$. For the second harmonic ( $2 g$-type), we observed no actual resonators in the region of $2 g-3 g_{5}+g_{6}$ and 116 resonators in the region of $2 g-3 g_{6}+g_{5}$. Finally, in the third harmonic ( $3 g$-type), we only found two resonators in the region of the $3 g-2 g_{5}-g_{6}$ resonance. In the other regions, we did not observe resonators, according to our identification criteria. Based on this analysis, we will focus our attention on the three $g$-type resonances that have a signif-

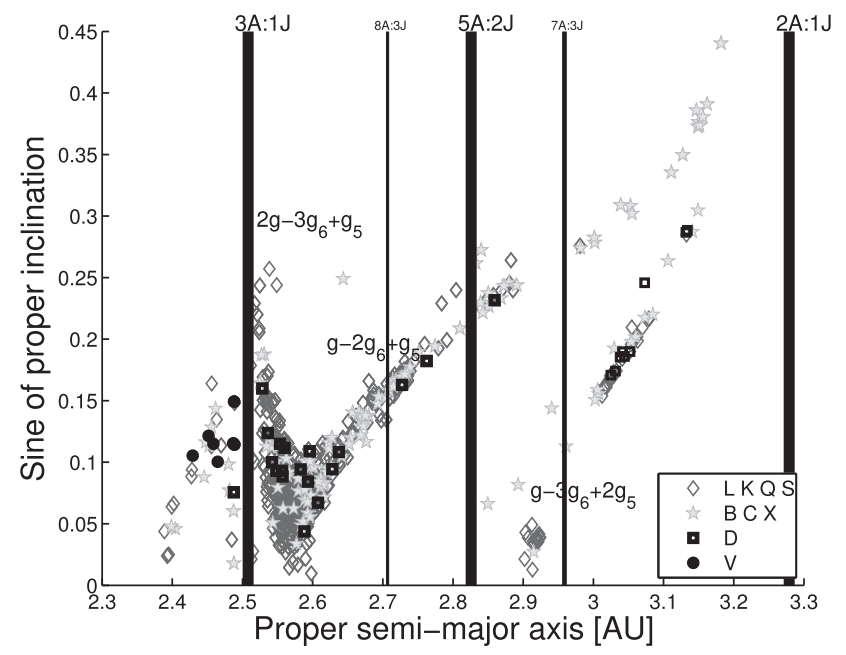

Figure 4. An $(a, \sin (i))$ projection of asteroids with taxonomical information in the regions of the $g-2 g_{6}+g_{5}, g-3 g_{6}+2 g_{5}$, and $2 g-3 g_{6}+g_{5}$ resonances.

icant number of actual resonators, the $g-2 g_{6}+g_{5}, g-3 g_{6}+2 g_{5}$ and $2 g-3 g_{6}+g_{5}$ resonances.

In the next subsection, we will analyse the taxonomic properties of asteroids in $g$-type secular resonant states.

\section{TAXONOMY OF ACTUAL RESONATORS}

To investigate the taxonomy of the identified actual resonators, we used the classification method of DeMeo \& Carry (2013), that employs data from Sloan Digital Sky Survey-Moving Object Catalog data, fourth release (SDSS-MOC4 hereafter, Ivezic et al. (2001)) to compute grid slope and $z^{\prime}-i^{\prime}$ colours and identify taxonomic properties of asteroids in this domain. In the region of $g-2 g_{6}+g_{5}$ resonance, we obtained a set of 1015 observations of asteroids (including multiple observations of the same object). This corresponds to 557 asteroids for which an SDSS-MOC4 taxonomical classification and proper elements are both available. We found $287 \mathrm{~S}, 98 \mathrm{~L}$, 46 C, 52 X, 19 D, 3 B, 2 Q, $47 \mathrm{~K}$ and 3 V type objects, respectively.

For the $g-3 g_{6}+2 g_{5}$ resonant region, we identified 149 observations of asteroids. These correspond to 92 asteroids with taxonomic types of which 14 are S, 19 L, 14 C, 20 X, 9 D, 2 B and 14 K types objects. Finally for the case of the $2 g-3 g_{6}+g_{5}$ resonance, we found 58 observations to 26 asteroids, divided as $12 \mathrm{~S}, 1 \mathrm{~L}, 5 \mathrm{C}, 4$ $\mathrm{X}$ and $4 \mathrm{~V}$ types.

Fig. 4 displays an $(a, \sin (i))$ projection of asteroids with taxonomic information in the three resonances. The identified bodies are displayed by different symbols according to their taxonomy: L-, K-, Q- and S-types are displayed with marked diamonds; B-, C- and $\mathrm{X}$-types are shown with marked stars, D-types with marked squares and V-types with black circles.

Our statistical analysis showed a greater amount of S-types in the region of $g-2 g_{6}+g_{5}$ resonance, near the 3J:-1A meanmotion resonance. Moreover, objects of C-and X-types are scattered in the region of $g-2 g_{6}+g_{5}$ and $g-3 g_{6}+2 g_{5}$ resonance, which are found in the outer main belt. The predominance of Stype in the inner main belt and of primitive types in the outer belt is in agreement with well-known distributions of these taxonomies in the main belt (Carruba et al. 2013).

Local inhomogeneities in the taxonomy of objects, i.e. primitive types in the inner main belt and S-complex objects in the outer main belt, can be caused by scattering of nearby asteroid families or 
Table 2. Families affected by $g$ - type non-linear secular resonances in the main belt, according to the data base from AstDys2. We report the FIN for the families for which this number is available in column 2.

\begin{tabular}{|c|c|c|c|c|c|}
\hline $\begin{array}{l}\text { Resonance argument } \\
\text { proper frequencies }\end{array}$ & $\begin{array}{l}\text { Id and FIN } \\
\text { family }\end{array}$ & $\begin{array}{l}\text { Members inside of } \\
\text { resonance argument }\end{array}$ & $\begin{array}{l}\text { Likely } \\
\text { resonator }\end{array}$ & $\begin{array}{l}\text { Orbits switching } \\
\text { L and C }\end{array}$ & $\begin{array}{l}\text { Actual } \\
\text { resonator }\end{array}$ \\
\hline \multirow{12}{*}{$g-2 g_{6}+g_{5}$} & Astraea & 2425 & 565 & 108 & 388 \\
\hline & Bankakuko & 53 & 19 & 4 & 12 \\
\hline & Ikenozenni & 88 & 18 & 6 & 10 \\
\hline & Wagman & 92 & 18 & 7 & 8 \\
\hline & 1999 AZ8 & 65 & 8 & - & 7 \\
\hline & Brasilia (FIN 608) & 4 & 4 & 1 & 3 \\
\hline & $2000 \mathrm{HC} 81$ & 49 & 8 & 2 & 2 \\
\hline & Gefion (FIN 516) & 5 & 3 & 2 & 1 \\
\hline & Eunomia (FIN 502) & 3 & 2 & 1 & - \\
\hline & Euphrosyne (FIN 901) & 5 & 5 & 2 & - \\
\hline & Koronis (FIN 605) & 189 & 84 & 7 & 20 \\
\hline & Telramud (FIN 614) & 13 & 6 & - & 2 \\
\hline \multirow{4}{*}{$g-3 g_{6}+2 g_{5}$} & Eos (FIN 606) & 219 & 136 & 39 & 14 \\
\hline & Ursula (FIN 631) & 8 & 7 & 1 & 2 \\
\hline & Tirela (FIN 612) & 17 & 11 & 1 & 1 \\
\hline & Euphrosyne (FIN 901) & 4 & 4 & 4 & - \\
\hline \multirow{9}{*}{$2 g-3 g_{6}+g_{5}$} & Vesta (FIN 401) & 85 & 21 & - & 7 \\
\hline & Massallia (FIN 404) & 385 & 47 & - & 13 \\
\hline & Nysa/Polana (FIN 405) & 727 & 158 & - & 17 \\
\hline & Sulamitis (FIN 408) & 42 & 15 & - & 1 \\
\hline & Eunomia (FIN 502) & 339 & 216 & 7 & 5 \\
\hline & Maria (FIN 506) & 44 & 27 & - & - \\
\hline & Euphrosyne (FIN 901) & 1 & 1 & - & - \\
\hline & Clarissa (FIN 407) & 21 & - & - & - \\
\hline & Erigone (FIN 163) & 17 & - & - & - \\
\hline
\end{tabular}

background objects. The next section will further investigate how local families are affected by these three $g$-type secular resonances.

\section{FAMILIES AFFECTED BY $g$-TYPE NON-LINEAR SECULAR RESONANCES}

We will now focus our attention on the asteroid families that are most affected by g-type non-linear secular resonances. Full statistical details of asteroid families in these resonant regions are displayed in Table 2, where we used the families identified by Nesvorný et al. (2015) and Milani et al. (2014), using the hierarchical clustering method (HCM) of Bendjoya \& Zappala (2002). Families that satisfied the selection criteria of Nesvorný et al. (2015) and are therefore considered to be more established are also identified by the FIN. The reader may notice that three families have a large number of members in the $g-2 g_{6}+g_{5}$ secular resonance: Astraea with 388 actual resonators, Ikenozenni with 10 actual resonators and Bankakuko with 12 actual resonators. Also, we have a small number of likely resonators for the Wagman family and Minerva family in the anti-aligned librating state (more details in Table A1). For the region of $g-3 g_{6}+2 g_{5}$ resonance, we have 20 actual resonators in the Koronis family and 14 actual resonators in the Eos family as reported in Table 2 . None of the families interacting with the $2 g-3 g_{6}+g_{5}$ secular resonance has more than 20 members in resonant states. For this reason, we will focus our analysis on asteroid families interacting with the $g-2 g_{6}+g_{5}$ and $g-3 g_{6}+2 g_{5}$ resonances.

We focus our attention on three asteroid families: the Astraea family that has the largest number of members in librating states of the $g-2 g_{6}+g_{5}$ resonance in the central main belt, and is cut by this resonance; the Brasilia family that is located on the right side and close to the $g-2 g_{6}+g_{5}$ resonant region; and finally, the Tirela group that is located on the left side of the resonant region of the $g-2 g_{6}+g_{5}$ resonance and that has members in librating states of the $g-3 g_{6}+2 g_{5}$ resonance. By considering these three families, we believe that we may be able to study how families were cut, and on the right and left side of the $g-2 g_{6}+g_{5}$ resonance evolve under the influence of this resonance.

We considered the families as identified in Nesvorný et al. (2015), that used HCM, and cutoff of $45 \mathrm{~m} \mathrm{~s}^{-1}$ (2477 members) for the Astraea group, $50 \mathrm{~m} \mathrm{~s}^{-1}$ (597 members) for the Brasilia group, and $50 \mathrm{~m} \mathrm{~s}^{-1}$ (1472 members) for the Tirela group, respectively. Also, we selected the maximum and minimum values of proper elements for each family (semimajor axis $a \pm 0.01$ au, eccentricity $e \pm$ 0.01 and sine of inclination $\sin (i) \pm 0.01)$, so as to define local background regions. Finally, we revised the physical properties of the three families with the methods in Section 5 and using the Widefield Infrared Survey Explorer (WISE) data base by Masiero et al. (2012) for information on geometric albedo and asteroid diameters.

\subsection{The Astraea group}

There were 9615 objects in the local background of the Astraea family, 690 of which with a spectral type. $54.3,16,5.2$ and 0.3 per cent of the total were S-type, L-type, X-type and V-type (these last two asteroids 119360 and 88084), respectively. Amongst Astraea family members, there were 193 asteroids (88 of which are $g-2 g_{6}+g_{5}$ resonators) with taxonomic information. 14 were $\mathrm{C}-, 1 \mathrm{~B}-, 8 \mathrm{D}-$, $17 \mathrm{~K}-, 27 \mathrm{~L}-, 12 \mathrm{X}$ - and 114 S-types. We also note that 54 out of the 114 S-type are $g-2 g_{6}+g_{5}$ resonators. Overall, the Astraea region is dominated by the S-complex asteroids. Concerning to albedo information, we identified 286 asteroids (109 were resonators) as members of the Astraea family. Our analysis confirms a predominance of high albedos, in a range from 0.0362 to 0.6288 . 


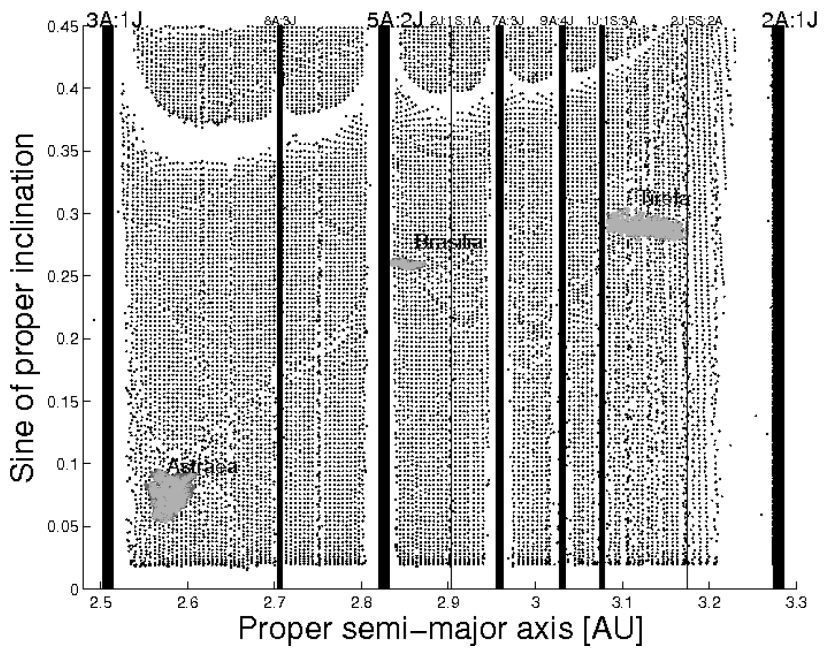

Figure 5. Projection in the $(a, \sin (i))$ plane of the Astraea, Brasilia and Tirela families. These dynamical groups are affected by the $g-2 g_{6}+g_{5}$ and $g-3 g_{6}+2 g_{5}$ non-linear secular resonances.

94 asteroids (32.8 per cent of the total) have $p_{V}<0.12$, associated with CX-complex objects, 128 (44.5 per cent of the total) bodies have $0.12<p_{V}<0.3$, associated with $\mathrm{S}$-complex taxonomies, and 64 asteroids ( 13.5 per cent of the total) have $p_{V}>0.3$, and are possible albedos interlopers. The average albedo of the Astraea family is $p_{V}=0.2039$, while family members in resonant states have an average albedo of $p_{V}=0.2465$.

\subsection{The Brasilia family}

In this family, there were 696 objects in the background, but only 49 objects with taxonomic information. 69.4 per cent of them were $\mathrm{X}$-type and 18.4 per cent $\mathrm{C}$-type, and the rest belonged to $\mathrm{S}$-complex classes. Of the 43 family members with an identified taxonomy, there were $6 \mathrm{C}, 2 \mathrm{~K}, 2 \mathrm{~L}, 2 \mathrm{~S}, 31 \mathrm{X}$-type, respectively. We found 116 objects in the WISE data base, the majority of which (96) with albedos in the S-complex range. While most of the Brasilia members are primitive bodies, they seem to be characterized by higher values of albedos than what are usually found for these objects: the average albedo value for this family was $p_{v}=0.1776$.

\subsection{The Tirela family}

3008 objects are found in the background of this family, 243 of which with taxonomical information. 35.8 per cent of them are D-type, 35.8 per cent L-type and 13.9 per cent C-types, with the rest belonging to other classes. Of the 121 members of the Tirela family with taxonomical information, we found $3 \mathrm{C}-, 53 \mathrm{D}-, 2 \mathrm{~K}-$ and 48 L-types, and eight questionable cases (with multiple observations). A total of 320 members of the family have WISE albedo data, the majority of which (217 objects) are associated with an S-complex taxonomy. The average albedo for the Tirela family is $p_{v}=0.2086$. As in Nesvorný et al. (2015), we consider the Tirela family to belong to an S-complex taxonomy, and treat primitive objects as interlopers.

\section{FAMILY AGE ESTIMATE}

In Fig. 5, we display our three families in our dynamical map in the $(a, \sin (i))$ plane. The Astraea family $(2477$ members $)$ in the

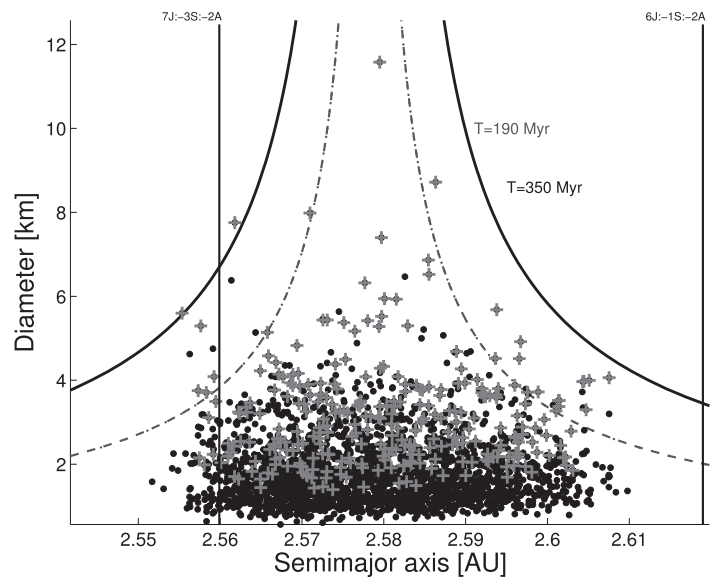

Figure 6. A proper $a$ versus diameter projection of members of the (5) Astraea family (black dots). Grey crosses display asteroids with taxonomical information in the region. The black and grey curves show isolines of the maximum and minimum displacement in $a$ caused by the Yarkovsky effect starting from the family barycenter.

central main belt, between the mean-motion resonances $3 \mathrm{~J}: 1 \mathrm{~A}$ and 2J:-2S:-1A, the Brasilia family (597 members) in the pristine region near the 5J:-2A mean-motion resonance and the Tirela family (1472 members) in the outer main belt, between $11 \mathrm{~J}:-5 \mathrm{~A}$ and $5 \mathrm{~J}:-2 \mathrm{~S}:-2 \mathrm{~A}$ mean-motion resonances.

The next step was to apply the method of Yarkovsky isolines to obtain a preliminary estimate of the ages of the Astraea, Brasilia and Tirela families. Since this method does not account for the initial dispersion of family members at the time of family formation, ages obtained with this approach should be considered just as upper limits of the real values. We used the estimated diameters from the WISE data base, when available, otherwise diameters were estimated using the absolute magnitudes and the mean value of geometric albedo ${ }^{3}$ in the orbital region of the family according to equation (1). We calculated the barycentre of the family in proper $a$, and the total mass of the family with or without the presumed parent body with the method described in Carruba (2011). We then used standard values of the parameters affecting the Yarkovsky force for S- and C-complex families. In particular, for the Astraea family we used thermal conductivity $K=0.001 \mathrm{~W} \mathrm{~m}^{-1} \mathrm{~K}^{-1}$, thermal capacity $C=$ $680 \mathrm{~J} \mathrm{~kg}^{-1} \mathrm{~K}^{-1}$, surface density equal to $1500 \mathrm{~kg} / \mathrm{m}^{-3}$, Bond albedo equal to 0.1 , thermal emissivity equal to 0.9 and bulk density equal to $2500 \mathrm{~kg} \mathrm{~m}^{-3}$. For the Brasilia and Tirela families we employed $K=0.01 \mathrm{~W} \mathrm{~m}^{-1} \mathrm{~K}^{-1}$, thermal capacity $C=680 \mathrm{~J} \mathrm{~kg}^{-1} \mathrm{~K}^{-1}$, surface density equal to $1500 \mathrm{~kg} / \mathrm{m}^{-3}$, Bond albedo equal to 0.02 , thermal emissivity equal to 0.95 and bulk density equal to $1500 \mathrm{~kg} \mathrm{~m}^{-3}$. See Brož et al. (2013) for further details on the choice of these parameters.

Note that for the family of Astraea, 97 per cent of the bodies had diameters less than $4 \mathrm{~km}$, while (5) Astraea itself has $D=115 \mathrm{~km}$, which is typical of a family formed in a cratering event. According to Nesvorný et al. (2015), Brasilia and Tirela originated in a scenario of fragmentation of the parent body.

Fig. 6. displays the results of our method for the Astraea family, the largest group in the region. For brevity, we do not show our

\footnotetext{
${ }^{3}$ While it is generally better to use the median value of the geometric albedo, so as to reduce the influence of possible interlopers, since the population of the studied groups is large enough, in this work we prefer to use the mean value.
} 
results for the Brasilia and Tirela families. We computed Yarkovsky isolines with values maximum of $350 \mathrm{Myr}$ and a minimum of 190 Myr, the estimated family age range according to Nesvorný et al. (2015). Our results for Brasilia yield a maximum of $260 \mathrm{Myr}$ and a minimum of $180 \mathrm{Myr}$. Finally, Tirela age is in a range from 600 to 690 Myr.

\section{DYNAMICAL EVOLUTION OF THE FAMILIES}

To investigate the dynamic evolution of the families in these resonant regions we used SWIFT-RMVSY, the symplectic integrator of Brož (1999) that simulates the diurnal and seasonal versions of the Yarkovsky effect. All planets from Venus to Neptune (Mercury was accounted for as a barycentric correction in the initial conditions) were accounted for, and the optimal values of the Yarkovsky parameters discussed in the previous section for S- and C-complex asteroids were used in our simulations.

We selected members of the families with absolute magnitude less than 15.5 , so as to concentrate on larger objects less likely to skip the studied resonances because of their slower Yarkovsky mobility. We also generated fictitious asteroid families (clones of asteroids for the HCM families obtained) to investigate the maximum possible diffusion of asteroids over $400 \mathrm{Myr}$ for Astraea and Brasilia, but 1200 Myr for Tirela, these time-scales comparable with the maximum estimated ages of the three families. ${ }^{4}$

We used two sets of spin axis orientations with \pm 90 with respect to the orbital plane, since these obliquities maximize the speed of the Yarkovsky effect. We assumed periods obtained under the approximation that the rotation frequency is inversely proportional to the object radius (Brož 1999). No re-orientations were considered, and we obtained synthetic proper elements with the approach described in Carruba (2010).

We then monitored the diffusion of family members in each local region, as defined in previous sections of this paper. For this purpose, we computed the fraction of objects that remained in the $(a, e, \sin (i))$ boxes previously defined. Fig. 7, Panel A, displays the time evolution of the fraction of all asteroids surviving in the Astraea family region, defined as a box (black curve). The fraction of surviving resonators is shown as a grey line. 40 per cent of the initial population survived, but a large fraction of the resonators was ejected. Panel B of the same figure presents an $(a, e)$ projection of the local background of the Astraea family, with proper elements of the test particles at the beginning and end of the simulation. The black lines identify local mean-motion resonances in this region. The dotted line identifies the region affected by the $g-2 g_{6}+g_{5}$ secular resonance. The centre of this resonance was computed using the FREK.F code, based on the perturbation model from Milani \&

\footnotetext{
${ }^{4}$ We should caution the reader that this approach may lead to either underestimating or overestimating the family age. The fictitious family initial spreads depend on the ejection speed from the parent body and on the values of $f$ and $\omega+f$ of the parent body at the moment of break-up. Depending on the used values of these parameters, the synthetic family could be either larger (as it could be the case for the Astraea group) or quite smaller than the current one. A detailed study of the optimal values of the ejection velocity field for the three groups here studied could be carried out, but it is beyond the purpose of this work, which aims at studying the effect that dynamical dispersion in $g$-type secular resonances may have had on the evolution of asteroid groups. More detailed studies of the optimal ages and initial ejection velocity field of these and other groups affected by these resonances are left as challenges for future research.
}

Kneževic (1994), to compute the proper frequencies $g$ and $s$ for values of $a$ and $e$ in the range shown in the figure, and fixing the inclination at $4^{\circ}$ (left curve) and $8^{\circ}$ (right curve), the minimum and maximum values of the inclination observed for our test particles at the end of the simulation. The reader can clearly notice the striking effect of the $g-2 g_{6}+g_{5}$ secular resonance in affecting the shape of the Astraea family, and how the final shape follows the $g-2 g_{6}+g_{5}$ resonance centres.

We repeated our analysis for the Brasilia and Tirela families, and our results are shown in panels $\mathrm{C}, \mathrm{D}, \mathrm{E}$ and $\mathrm{F}$. The centre of the $g-2 g_{6}+g_{5}$ was computed for $i=12.5$ and 13.6 for the Brasilia family, and for $i=13.0$ and 21.0 for the Tirela group.

In the case of the Brasilia family, the resonance itself plays a minor role, but, by injecting asteroids in the $5 \mathrm{~A}: 2 \mathrm{~J}$ mean-motion resonance, it helps increasing the diffusion caused by dynamical mechanism of mobility. The fraction of resonant objects surviving in the region significantly decreases as a function of time for this region. The Tirela family is located on the left side of the $g-2 g_{6}+g_{5}$ resonance and on the right side of the $2 g-3 g_{6}+2 g_{5}$ resonance, in semimajor axis. The net effect of these resonances is to increase the spread in eccentricity of the family and to help the diffusion of members caused by the nearby $2 A: 1 J$ mean-motion resonance.

To quantify the effect on proper $e$ and $\sin (i)$ caused by the local secular resonances, we calculated the time behaviour of the standard deviation of $\delta e$ and $\delta \sin (i)$ for all three families, according to equation 4:

$\delta K^{2}=\frac{1}{N(N-1)} \sum_{i \neq j}\left(K_{j}-K_{i}\right)$,

where $N$ is the number of integrated bodies for each family and $K$ is the proper elements $(e$ and $\sin i)$ of the $i$-th body $(i=1, \ldots, N)$. To better understand the effect of the $g-2 g_{6}+g_{5}$ resonance, we concentrate on the Brasilia and Tirela families, that are on the right and left sides of this resonance.

Fig. 8, Panel A, shows the standard deviation in proper $\delta e$ of 291 clones of asteroids in the region of the Brasilia family. The black curves refer to members of the Brasilia family, while the light grey dotted curves refer to resonators of the $g-2 g_{6}+g_{5}$ resonance. Similar results are shown for the Tirela family in Fig. 9. The median value of $\delta e$ for the Brasilia family was of 0.0015 , while the displacement in $\sin (i)$ was just 0.00038 . For Tirela Family, the median value of $\delta e$ and $\sin (i)$ are 0.0158 and 0.0065 , respectively. Overall, this analysis confirms the effectiveness of the $g-2 g_{6}+g_{5}$ in changing the eccentricity of asteroids in their region. As expected for a $g$-type resonance, long-term changes in the inclination tend to be negligible.

\section{CONCLUSIONS}

The main goal of this article was to study the main $g$-type non-linear secular resonances in the asteroid main belt. Our results could be summarized as follows:

(i) We studied the orbital regions affected by the main $g$-type nonlinear secular resonances of argument $g-2 g_{6}+g_{5}, g-3 g_{6}+2 g_{5}$ and $2 g-3 g_{5}+g_{6}$, and obtained the population of $H<15.5$ asteroids most likely to be affected by these resonances.

(ii) We revised the physical properties of likely resonators in the areas affected by these three resonances. Not surprisingly, resonant regions in the inner main belt show a predominance of S-complex asteroids, while resonant regions in the outer main belt are dominated by more primitive types. Local mixing of 

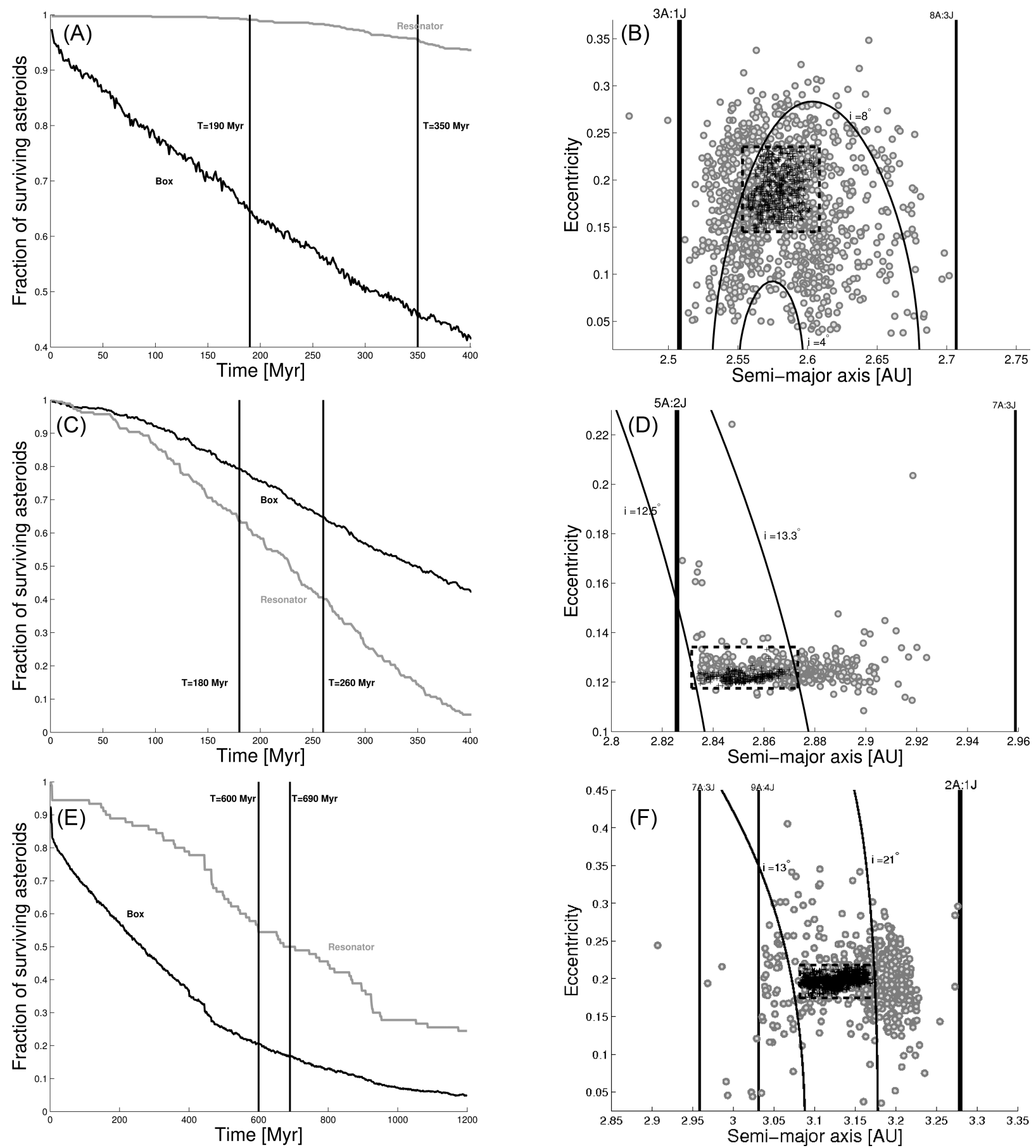

Figure 7. Panels A, C, E: fractions of initial population surviving, fraction of resonators, and fraction of objects remaining in the local background for the three studied families. Panels B, D and F: an $(a, e)$, projection of the final state of the simulated families at the end of our simulations.

other taxonomical types can be caused by nearby fonts, such as local families or objects in the local background of different spectral types.

(iii) We identified the number of asteroids in actual states of libration of these three and other $g$-type resonances. The largest number of resonant objects is observed in the $g-2 g_{6}+g_{5}$ resonance, followed by the $g-3 g_{5}+2 g_{6}$ resonance and, in a lesser measure, by the $2 g-3 g_{5}+g_{6}$ resonance. Amongst asteroid families affected by the $g-2 g_{6}+g_{5}$ resonance, the Astraea, the Ikenozenni and the Bankakuko families all have more than 50 per cent of their members in resonant states. No family was observed to have more than 20 per cent of their members in resonant states of the $g-3 g_{5}+2 g_{6}$ and $2 g-3 g_{5}+g_{6}$ resonances.

(iv) Three asteroid families are studied in this work, all characterized by their interaction with the $g-2 g_{6}+g_{5}$ resonance: the Astraea (cut by this resonance), the Brasilia (on the left side of this resonance) and the Tirela family (on the right side of the resonance in semimajor axis). The Astraea family was most likely formed in a cratering event, while the Brasilia and Tirela were probably the outcome of a fragmentation scenario. 


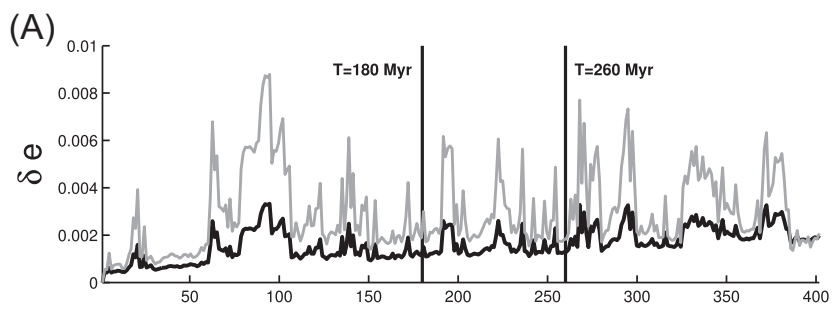

(B)

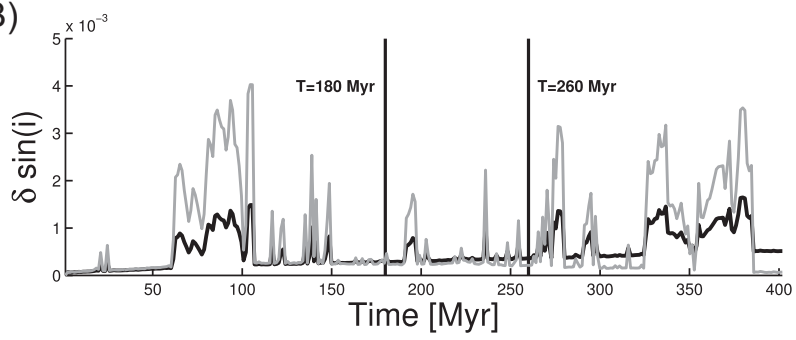

Figure 8. The time-evolution of the standard deviations in proper $\delta e$ (Panel A) and $\delta \sin (i)($ Panel B) for the 291 clones of the member of the Brasilia family in our simulation. The black curve refers to members of the Brasilia family, the grey curve refers to resonators of the $g-2 g_{6}+g_{5}$ resonance. Black vertical lines display the minimum lower and maximum upper limits for the family age, estimated using the Yarkovsky isolines method.
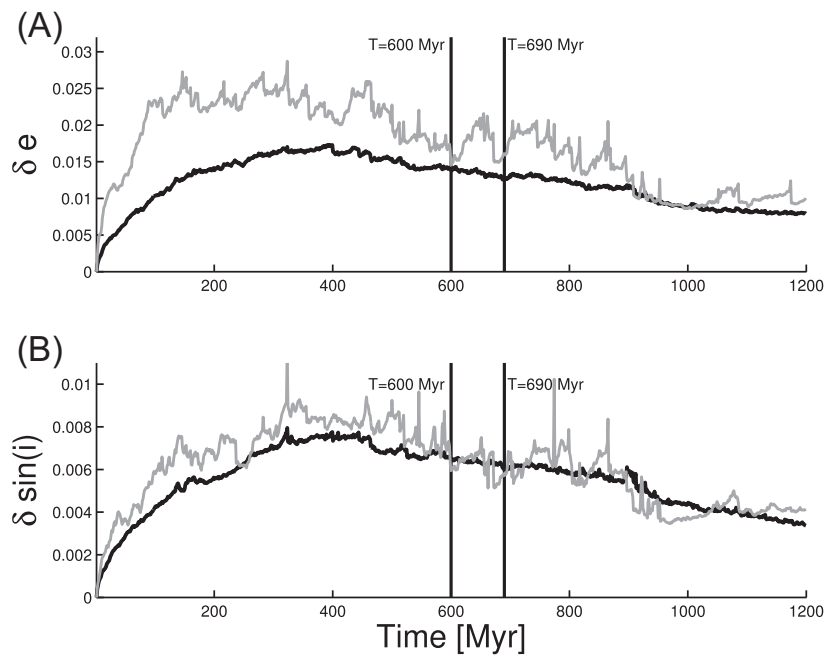

Figure 9. The time-evolution of the standard deviation in proper $\delta e$ (Panel A) and $\delta \sin (i)$ (Panel B) for 2046 clones of the member of the Tirela family. Symbols are the same as in Fig. 8.

(v) We estimated the family ages using the method of Yarkovsky isolines. Their nominal ages are in the range from 190 to $350 \mathrm{Myr}$ for Astraea, from 180 to $260 \mathrm{Myr}$ for Brasilia and from 600 to $690 \mathrm{Myr}$ for Tirela, respectively.

(vi) Over the estimated maximum ages of the families, changes in the standard deviation in proper $e$ for the Brasilia family were of the order of 0.0015 , while they were of the order of 0.0160 for Tirela. Changes in the standard deviation of $\sin (i)$ were essentially negligible. We confirm the effectiveness of the $g-2 g_{6}+g_{5}$ resonance in affecting the shape of families inside this resonance, such as the Astraea family.

Overall, our results show that $g$-type resonances can be effective in shaping families that are crossed by this type of resonances, and can increase their dynamical dispersion in the proper $(a, e, \sin (i))$ domain. As expected, the long-term effect on asteroid inclinations of this type of resonances is essentially negligible.

\section{ACKNOWLEDGEMENTS}

We are grateful to Dr Miroslav Brož for comments and suggestions that significantly improved the quality of this paper. The authors wish to thank the Sao Paulo State Science Foundation (FAPESP; grants 2016/04476 - 8 and 2013/15357 - 1) and the Brazilian National Research Council (CNPq; grants 305453/2011 - 4 and $310317 / 2016$ - 9) for the generous support of this work. This publication makes use of data products from the Wide-field Infrared Survey Explorer (WISE) and Near-Earth Object WISE (NEOWISE), which are a joint project of the University of California, Los Angeles, and the Jet Propulsion Laboratory/California Institute of Technology funded by the National Aeronautics and Space Administration.

\section{REFERENCES}

Bendjoya P., Zappala V., 2002, in Bottke W. F., Jr, Cellino A., Paolicchi P., Binzel R. P., eds, Asteroids III. University of Arizona Press, Tucson, AZ, p. 613

Brož M., Morbidelli A., Bottke W. F., Rozehnal J., Vokrouhlický D., Nesvorný D., 2013, A\&A, 551, A117

Brož M., 1999, PhD thesis, Charles Univ., Prague

Carruba V., 2009, MNRAS, 395, 358

Carruba V., 2010, MNRAS, 408, 580

Carruba V., 2011, MNRAS, 395, 358

Carruba V., Burns J. A., Bottke W., Nesvornỳ D., 2003, Icarus, 162, 308

Carruba V., Domingos R. C., Nesvorný D., Roig F., Huaman M. E., Souami D., 2013, MNRAS, 433, 2075

Charlier C. V. L., 1902, in Von Veit V., Leipzig C., eds, Die Mechanik des Himmels, p. 410. Available at: https://archive.org/details/ diemechanikdesh04chargoog

DeMeo F., Carry B., 2013, Icarus, 266, 723

Ivezic̀ Ž. et al., 2001, AJ, 122, 2749

Knežević Z., Milani A., 2003, A\&A, 403, 1165

Kneževic Z., Milani A., Farinella P., Froeschle Ch., Froeschle Cl., 1991, Icarus, 93, 316

Levison H. F., Duncan M. J., 1994, Icarus, 108, 18

Machuca J. F., Carruba V., 2012, MNRAS, 420, 1779

Masiero J. R., Mainzer A. K., Grav T., Bauer J. M., Jedicke R., 2012, ApJ, 759, 14

Milani A., Kneževic Z., 1994, Icarus, 107, 219

Milani A., Cellino A., Kneževic Z., Novaković B., Spoto F., Paolicchi P. 2014, Icarus, 239, 46

Morbidelli A., Henrard J., 1991, Celest. Mech., 51, 169

Nesvornỳ D., Brož M., Carruba V., 2015, in Michel P., DeMeo F. E., Bottke W., eds, Asteroid IV. Univ. Arizona Press, Tucson, AZ, p. 297

Williams J. G., 1969, PhD Dissertation, Univ. California, Los Angeles

\section{APPENDIX: TABLES}

Statistical analysis of likely resonators in the resonant region of the $g$-type non-linear secular resonance in the main belt. We provide the resonant argument in terms of proper frequencies, the maximum absolute magnitude considered, and the number of objects that are likely resonators, on circulating orbits, on orbits alternating phases of circulation and libration, and on librating orbits. 
Table A1. Analysis of likely resonators in the resonant region of the $g$-type non-linear secular resonance in the main belt.

\begin{tabular}{lccccc}
\hline $\begin{array}{l}\text { Resonance argument } \\
\text { proper frequencies }\end{array}$ & $\begin{array}{c}\text { Magnitude } \\
H\end{array}$ & $\begin{array}{c}\text { Likely } \\
\text { resonators }\end{array}$ & $\begin{array}{c}\text { Circulation } \\
(\mathrm{C})\end{array}$ & $\begin{array}{c}\text { Switching orbits } \\
\text { L and C }\end{array}$ & $\begin{array}{c}\text { Libration } \\
(\mathrm{L})\end{array}$ \\
\hline & $<13.5$ & 197 & 61 & 72 & 64 \\
$g-2 g_{6}+g_{5}$ & $<14.5$ & 864 & 248 & 363 & 253 \\
& $<15.5$ & 3293 & 825 & 951 & 1517 \\
$g-3 g_{6}+2 g_{5}$ & $<15.5$ & 694 & 371 & 102 & 128 \\
$2 g-3 g_{6}+g_{5}$ & $<15.5$ & 1178 & 770 & 46 & 116 \\
\hline
\end{tabular}

This paper has been typeset from a $\mathrm{T}_{\mathrm{E}} \mathrm{X} / \mathrm{L} \mathrm{T} \mathrm{E} X$ file prepared by the author. 